\title{
The Regge Limit and infrared singularities of QCD scattering amplitudes to all orders
}

\author{
Leonardo Vernazza* \\ Nikhef, Science Park 105, NL-1098 XG Amsterdam, The Netherlands \\ E-mail: 1.vernazza@nikhef.nl
}

\section{Simon Caron-Huot}

Department of Physics, McGill University, 3600 rue University, Montréal, QC H3A 2T8, Canada

E-mail: schuotephysics.mcgill.ca

\section{Einan Gardi}

Higgs Centre for Theoretical Physics, School of Physics and Astronomy, The University of Edinburgh, James Clerk Maxwell Building, Peter Guthrie Tait Road, Edinburgh EH9 3FD, Scotland, U.K.

E-mail: Einan.Gardi@ed.ac.uk

\section{Joscha Reichel}

Higgs Centre for Theoretical Physics, School of Physics and Astronomy, The University of Edinburgh, James Clerk Maxwell Building, Peter Guthrie Tait Road, Edinburgh EH9 3FD,

Scotland, U.K.

E-mail: joscha.reichel@ed.ac.uk

In this talk we discuss recent progress in understanding the iterative structure of scattering amplitudes in the high-energy limit. As an application, we show that by using BFKL theory it is possible to determine the infrared divergent part of two-parton amplitudes to all orders in perturbation theory and to next-to-leading-logarithmic accuracy in the high-energy limit, resumming the two-Reggeon contribution. Remarkably, the infrared-singular part can be expressed in a closed form. This allows us to predict the Regge limit of the soft anomalous dimension to any loop order.

Loops and Legs in Quantum Field Theory (LL2018)

29 April 2018 - 04 May 2018

St. Goar, Germany

\footnotetext{
*Speaker.
} 


\section{Introduction}

The high-energy limit of gauge-theory scattering amplitudes offers a unique insight into gauge dynamics. In this kinematic limit, in which the centre of mass energy $s$ is much larger than the momentum transfer $-t$, amplitudes simplify and factorise in rapidity, giving rise to new degrees of freedom in two dimensions. Within perturbative QCD, BFKL $[1,2]$ and related rapidity evolution equations allow us to translate concepts from Regge theory [3] into calculation tools, leading to concrete predictions.

The simplest example is that of the reggeized gluon, the effective interaction which governs the behaviour of two partons scattering amplitudes in QCD. In the leading logarithmic approximation the exchange of a single reggeized gluon leads to a trivial evolution equation in rapidity, which amounts to straightforward exponentiation of logarithms of $|s / t|$ to all orders in the coupling. This is manifestation of a Regge pole. At higher logarithmic accuracy a more complex analytic structure emerges, which can be understood in QCD as compound states of two or more reggeized gluons, which form Regge cuts [5, 6, 7]. In contrast to the single Reggeon case, the corresponding evolution equations are difficult to solve in general $[8,9]$. Nevertheless, they can be integrated iteratively, thus generating perturbative high-energy amplitudes order-by-order in the coupling $[11,12]$.

The factorisation structure of two-parton scattering in the high-energy limit is best studied by decomposing the amplitude into its odd and even components with respect to $s \leftrightarrow u$ exchange, the so-called signature:

$$
\mathscr{M}^{( \pm)}(s, t)=\frac{1}{2}(\mathscr{M}(s, t) \pm \mathscr{M}(-s-t, t))
$$

In case of two-gluon scattering, which will be the focus of this talk, this decomposition is particularly useful, because, due to Bose symmetry, the symmetry properties under the exchange $s \leftrightarrow u$ are mirrored into the color structure of the amplitude. The latter can be seen as a vector in color space, and decomposed onto an orthonormal color basis defined by the colour flow in the $t$ channel. For example, in the case of $g g \rightarrow g g$ scattering the decomposition is

$$
8 \otimes 8=1 \oplus 8_{s} \oplus 8_{a} \oplus 10 \oplus \overline{10} \oplus 27,
$$

where the numbers label the color representations by their dimension in SU(3). The amplitudes $\mathscr{M}^{[1]}, \mathscr{M}^{\left[8_{s}\right]}$ and $\mathscr{M}^{[27]}$ are thus even, while the amplitudes $\mathscr{M}^{\left[8_{a}\right]}$ and $\mathscr{M}^{[10+\overline{10}]}$ are odd under $s \leftrightarrow u$ exchange.

The signature-odd amplitude is governed by an odd number of reggeized gluons, thus, up to next-to-leading logarithmic accuracy, the scattering process occurs by means of a single reggeized gluon exchange (only $8_{s}$ in (1.2) is contributing). The amplitude is obtained by dressing the $t$ channel gluon propagator by a power of $s / t$, known as Regge trajectory, which captures the dependence on the energy, and energy-independent impact factors, that depend on the colliding partons (first two diagrams in the left column of figure 1). The effect of multiple Reggeon exchange starts in the odd amplitude only at next-to-next-to logarithmic accuracy. This was recently demonstrated explicitly in ref. [12], where these effects were computed through three-loops, by constructing an iterative solution of the relevant Balitsky-JIMWLK equation describing the evolution of three reggeized gluons and their mixing with a single reggeized gluon. We refer to [12] (see also [14, 15]) for a detailed discussion. 


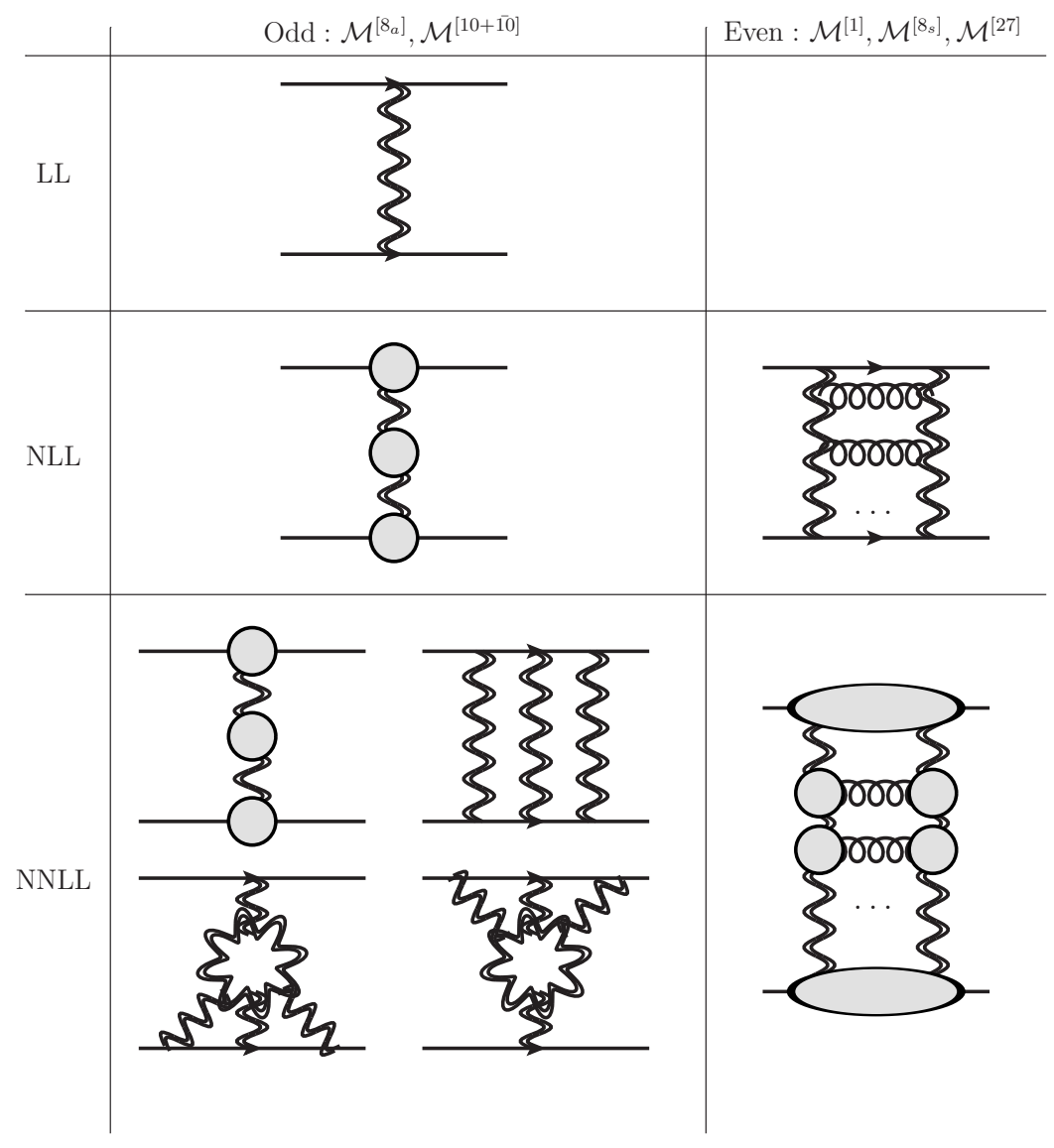

Figure 1: Structure of high-energy logarithms in two-gluon scattering amplitude.

In this talk we focus on the even part of the amplitude, which involves the exchange of an even number of reggeized gluons (right column in figure 1). The leading logarithmic corrections to the even amplitude are determined to all orders by a wavefunction of a pair of reggeized gluons, which solves the BFKL evolution equation. This iterative solution, discussed in what follows, is described by ladder graphs, where an additional rung is generated at each order in the loop expansion.

The study of the even amplitude in the high-energy limit has an important application in relation to the problem of determining the structure of infrared divergences of a scattering amplitude. It is well known that gauge-theory amplitudes have long-distance singularities, which cancel in physical observables such as sufficiently inclusive cross sections. These singularities are largely process-independent, and admit evolution equations leading to exponentiation. Of special interest are soft singularities, which in contrast to collinear ones, are sensitive to the colour flow of the underlying hard process. Soft singularities can be computed by considering correlators of semi-infinite Wilson lines. The corresponding soft anomalous dimension encodes the structure of these singularities to all orders in perturbation theory. In recent years there has been significant progress $[19,20,21,22,23,24]$ in determining the precise structure of long-distance singularities to massless gauge theories. Recently, an explicit computation has determined the soft anomalous 
dimension to three loops, for any number of external legs $[25,26]$. A remarkable feature is that infrared singularities are governed through two loops by a sum over colour dipoles formed by pairs of the hard-scattered partons; only starting at three loops one encounters infrared singularities that are simultaneously sensitive to the colour and kinematics of three and four hard partons.

While infrared factorization of fixed-angle scattering and high-energy factorization start from different kinematic setups, their interplay is well known [17, 16, 18, 24, 11]. The gluon Regge trajectory is infrared-singular, and its exponentiation along with the energy logarithms is consistent with the exponentiation of soft singularities through the relevant renormalization group equation. The correspondence between the structure of amplitudes in the high-energy limit, which is governed by rapidity evolution equations, and the structure of infrared singularities becomes more complicated at subleading orders. While both separately provide means to explore the structure of amplitudes to all orders in perturbation theory, the interplay between the two provides additional insight in both directions. For instance, Ref. [11] used the BFKL equation to compute the first few orders in the Regge cut of the signature even part of the amplitude at NLL accuracy, and predicted a corresponding correction to the soft anomalous dimension in the high-energy limit at four loops.

Motivated by this result, in Ref. [13] we have studied the wavefunction of a pair of reggeized gluons, which solves the BFKL evolution equation. The BFKL Hamiltonian has been diagonalised in the literature, see e.g. [4]. However, the study of partonic amplitudes requires us to use the dimensionally-regulated Hamiltonian, which is comparatively less understood. In this talk we will focus on the main result of Ref. [13], namely, the fact that it is nonetheless possible to find an exact iterative solution, based on the following two reasons: the two-Reggeon wavefunction itself turns out to be finite at all orders, so that infrared divergences are controlled by the limit of the wavefunction where a reggeized gluon becomes soft. The evolution equation then closes within that limit, dramatically simplifying its solution. This enables us to obtain the soft limit of the two-Reggeon wavefunction to all loop orders and NLL accuracy, and corresponding closed-form expressions for the singular part of the amplitude and soft anomalous dimension, which turns out to be an entire function of the coupling. The result is particularly interesting in the light of recent work, [27], which has shown that the functional form of the three-loop soft anomalous dimension in general kinematics can in fact be fully recovered via a bootstrap procedure using the high-energy limit of two parton scattering, alongside with other information, as input. The bootstrap programme of the soft anomalous dimension can be extended beyond three loops, provided that information from special kinematic limits is available, and our result in [13] provides data for this analysis for the first time to all orders in perturbation theory.

\section{The two-Reggeon cut}

Let us consider a $2 \rightarrow 2$ scattering amplitude $\mathscr{M}_{i j \rightarrow i j}$, where $i, j$ can be a quark or a gluon. The high-energy limit corresponds to a configuration of forward scattering, such that the Mandelstam variables satisfy $s \gg-t>0$. In the following we will focus on the even amplitude $\mathscr{M}_{+}$introduced in eq. (1.1), and express it as a function of the natural signature-even combination of logarithms

$$
\frac{1}{2}\left(\log \frac{-s-i 0}{-t}+\log \frac{-u-i 0}{-t}\right) \simeq \log \left|\frac{s}{t}\right|-i \frac{\pi}{2} \equiv L
$$


We discuss an effect that originates from the exchange of two Reggeons, therefore it is useful to remove the one-Reggeon exchange, defining a reduced amplitude,

$$
\hat{\mathscr{M}}_{i j \rightarrow i j} \equiv e^{-\mathbf{T}_{t}^{2} \alpha_{g}(t) L} \mathscr{M}_{i j \rightarrow i j},
$$

where $\mathbf{T}_{t}^{2}$ represents the total colour charge exchanged in the $t$ channel, and the function $\alpha_{g}(t)$ represents the gluon Regge trajectory.

The leading contributions to the signature-even amplitude, to all orders, corresponds to the two-Reggeon exchange. We denote it $\hat{\mathscr{M}}_{\mathrm{NLL}}^{(+)}$, given that it starts at next-to-leading logarithmic order compared to the single-Reggeon contribution to the odd amplitude $\mathscr{M}_{\mathrm{LL}}^{(-)} \sim e^{\mathbf{T}_{t}^{2} \alpha_{g}(t) L} \mathscr{M}^{(\text {tree })}$. The two-Reggeon exchange was studied long ago $[1,2]$ and can be expressed in terms the tworeggeized-gluon wavefunction $\Omega(p, k)$ as follows:

$$
\hat{\mathscr{M}}_{\mathrm{NLL}}^{(+)}\left(\frac{s}{-t}\right)=-i \pi \int[\mathrm{D} k] \frac{p^{2}}{k^{2}(p-k)^{2}} \Omega(p, k) \mathbf{T}_{s-u}^{2} \mathscr{M}_{i j \rightarrow i j}^{(\text {tree })},
$$

where $p^{2}=-t$, and the integration measure is

$$
[\mathrm{D} k] \equiv \frac{\pi}{B_{0}}\left(\frac{\mu^{2}}{4 \pi e^{-\gamma_{E}}}\right)^{\varepsilon} \frac{\mathrm{d}^{2-2 \varepsilon} k}{(2 \pi)^{2-2 \varepsilon}} .
$$

with $B_{0} \equiv B_{0}(\varepsilon)=e^{\varepsilon \varepsilon_{E}} \frac{\Gamma^{2}(1-\varepsilon) \Gamma(1+\varepsilon)}{\Gamma(1-2 \varepsilon)}=1-\frac{\zeta_{2}}{2} \varepsilon^{2}-\frac{7 \zeta_{3}}{3} \varepsilon^{3}+\ldots$, and the tree amplitude is

$$
\mathscr{M}_{i j \rightarrow i j}^{(\text {tree })}=4 \pi \alpha_{s} \frac{2 s}{t}\left(T_{i}^{b}\right)_{a_{1} a_{4}}\left(T_{j}^{b}\right)_{a_{2} a_{3}} \delta_{\lambda_{1} \lambda_{4}} \delta_{\lambda_{2} \lambda_{3}}
$$

where $\lambda_{i}$ for $i=1$ through 4 are helicity indices. The colour operator $\mathbf{T}_{s-u}^{2}$ in eq. (2.3) acts on $\mathscr{M}_{i j \rightarrow i j}^{\text {(tree) }}$ and it is defined in terms of the usual basis of Casimirs corresponding to colour flow through the three channels [10,18]: $\mathbf{T}_{s-u}^{2} \equiv\left(\mathbf{T}_{s}^{2}-\mathbf{T}_{u}^{2}\right) / 2$, with $\mathbf{T}_{s}=\mathbf{T}_{1}+\mathbf{T}_{2}=-\mathbf{T}_{3}-\mathbf{T}_{4}, \mathbf{T}_{u}=\mathbf{T}_{1}+\mathbf{T}_{3}=$ $-\mathbf{T}_{2}-\mathbf{T}_{4}, \mathbf{T}_{t}=\mathbf{T}_{1}+\mathbf{T}_{4}=-\mathbf{T}_{2}-\mathbf{T}_{3}$, where $\mathbf{T}_{i}$ represent the colour charge operators [19] in the representation corresponding to parton $i$. The wavefunction $\Omega(p, k)$ has a perturbative expansion in the strong coupling, taking the form

$$
\Omega(p, k)=\sum_{\ell=1}^{\infty}\left(\frac{\alpha_{s}}{\pi}\right)^{\ell} L^{\ell-1} \frac{B_{0}^{\ell}}{(\ell-1) !} \Omega^{(\ell-1)}(p, k),
$$

where we set the renormalization scale equal to the momentum transfer, $\mu^{2}=-t=p^{2}$. The amplitude itself is expanded as follows:

$$
\hat{\mathscr{M}}_{\mathrm{NLL}}^{(+)}\left(\frac{s}{-t}\right)=\sum_{\ell=1}^{\infty}\left(\frac{\alpha_{s}}{\pi}\right)^{\ell} L^{\ell-1} \hat{\mathscr{M}}_{\mathrm{NLL}}^{(+, \ell)} .
$$

$\hat{\mathscr{M}}_{\mathrm{NLL}}^{(+, \ell)}$ contains $\ell$-loop diagrams and can be computed from the $(\ell-1)$-loop contribution to the wavefunction through integration

$$
\hat{\mathscr{M}}_{\mathrm{NLL}}^{(+, \ell)}=-i \pi \frac{\left(B_{0}\right)^{\ell}}{(\ell-1) !} \int[\mathrm{D} k] \frac{p^{2}}{k^{2}(p-k)^{2}} \Omega^{(\ell-1)}(p, k) \mathbf{T}_{s-u}^{2} \mathscr{M}^{(\text {tree })} .
$$






Figure 2: Graphical representation of the amplitude at NLL accuracy, as obtained through BFKL evolution. The addition of one rung corresponds to applying once the leading-order BFKL evolution onto the wavefunction of order $(\ell-2)$. This gives the wavefunction at order $(\ell-1)$, according to eq. (2.9). Closing the ladder and integrating over the resulting loop momentum gives the reduced amplitude, according to eq. (2.8).

In the normalisation used in eq. (2.8), the leading-order wavefunction is simply $\Omega^{(0)}(p, k)=1$. At loop level the wavefunction is then obtained iteratively by applying the BFKL Hamiltonian:

$$
\begin{aligned}
\Omega^{(\ell-1)}(p, k) & =\left(2 C_{A}-\mathbf{T}_{t}^{2}\right) \int\left[\mathrm{D} k^{\prime}\right] f\left(p, k, k^{\prime}\right) \Omega^{(\ell-2)}\left(p, k^{\prime}\right)+\tilde{J}(p, k) \Omega^{(\ell-2)}(p, k) \\
& \equiv \hat{H} \Omega^{(\ell-2)}(p, k)
\end{aligned}
$$

where $f\left(p, k, k^{\prime}\right)$ is the BFKL evolution kernel

$$
f\left(p, k, k^{\prime}\right) \equiv \frac{k^{2}}{k^{\prime 2}\left(k-k^{\prime}\right)^{2}}+\frac{(p-k)^{2}}{\left(p-k^{\prime}\right)^{2}\left(k-k^{\prime}\right)^{2}}-\frac{p^{2}}{k^{\prime 2}\left(p-k^{\prime}\right)^{2}},
$$

and the function $\tilde{J}(p, k)$ is

$$
\tilde{J}(p, k)=\frac{1}{2 \varepsilon}\left[C_{A}\left(\frac{p^{2}}{k^{2}}\right)^{\varepsilon}+C_{A}\left(\frac{p^{2}}{(p-k)^{2}}\right)^{\varepsilon}-\mathbf{T}_{t}^{2}\right] .
$$

Eq. (2.9) is the standard BFKL Hamiltonian (see eq. (17) of the initial reference [1]) written using dimensional regularisation as an infrared regulator. $\tilde{J}(p, k)$ accounts for the Regge trajectories of the individual reggeized gluons, minus the overall Regge trajectory with colour charge $\mathbf{T}_{t}^{2}$ which was subtracted in the exponent of the reduced amplitude (2.2).

A graphical representation of eq. (2.8) is provided in figure 2. As a result of BFKL evolution, the amplitude at NLL accuracy can be represented as a ladder. At order $\ell$ it is obtained by closing the ladder and integrating the wavefunction of order $(\ell-1)$ over the resulting loop momentum, according to eq. (2.8). The wavefunction $\Omega^{(\ell-1)}(p, k)$, in turn, is obtained by applying once the leading-order BFKL evolution kernel to the wavefunction of order $(\ell-2)$. Graphically, this operation corresponds to adding one rung to the ladder.

Eq. (2.8) shows that the $\ell$-th order amplitude is obtained in terms of iterated integrals, which arise upon evaluating the wavefunction $\Omega^{(\ell-1)}(p, k)$ to order $(\ell-1)$. It is straightforward to compute the first few orders; our aim, however, is to determine the infrared singular part of the amplitude, in order to compare with the predictions made by the infrared factorisation theorem and, 
consequently, deduce higher-order corrections to the high-energy soft anomalous dimension. This goal can be achieved more easily by noticing that the wavefunction $\Omega^{(\ell-1)}(p, k)$ is finite for $\varepsilon \rightarrow 0$ to all orders in perturbation theory. This feature has practical implications, because it means that all divergences in the amplitude must originate in the final integration, namely going from the wavefunction to the amplitude as in eq. (2.3). Inspecting the latter equation, we see that divergences arise only in the $k \rightarrow 0$ and $k \rightarrow p$ limits. Due to the symmetry of the integrand, all divergences of the amplitude can therefore be obtained by evaluating it in one of these two limits, and multiplying the result by two. We consider here the the small- $k$ or soft limit $k \ll p$. It can be shown (see [13] for more details) that the evolution in eq. (2.9) reduces in this limit to $\Omega_{s}^{(\ell-1)}(p, k)=\hat{H}_{s} \Omega_{s}^{(\ell-2)}(p, k)$, with

$$
\hat{H}_{s} \Psi(p, k)=\left(2 C_{A}-\mathbf{T}_{t}^{2}\right) \int\left[\mathrm{D} k^{\prime}\right] \frac{2\left(k \cdot k^{\prime}\right)}{k^{\prime 2}\left(k-k^{\prime}\right)^{2}}\left[\Psi\left(p, k^{\prime}\right)-\Psi(p, k)\right]+\left(C_{A}-\mathbf{T}_{t}^{2}\right) J_{s}(p, k) \Psi(p, k),
$$

where

$$
J_{s}(p, k)=\frac{1}{2 \varepsilon}\left[1-\left(\frac{p^{2}}{k^{2}}\right)^{\varepsilon}\right] .
$$

Eq. (2.12) confirms that it is consistent to truncate the Regge evolution to the soft approximation: using the power counting $\Psi(p, k) \sim 1$, we see that the $k^{\prime}$ integral is saturated by the soft region $k^{\prime} \sim k$, with no sensitivity to larger scales.

Eq. (2.12) has a much simpler structure compared to the original one, because the soft approximation turns a two-scale problem into a one-scale problem. It is easy to check that the wavefunction reduces to a polynomial in $\xi=\left(p^{2} / k^{2}\right)^{\varepsilon}$, which implies that the integrals involved in eq. (2.12) are simple bubble integrals of the type

$$
\int\left[\mathrm{D} k^{\prime}\right] \frac{2\left(k \cdot k^{\prime}\right)}{k^{\prime 2}\left(k-k^{\prime}\right)^{2}}\left(\frac{p^{2}}{k^{\prime 2}}\right)^{n \varepsilon}=-\frac{1}{2 \varepsilon} \frac{B_{n}(\varepsilon)}{B_{0}(\varepsilon)}\left(\frac{p^{2}}{k^{2}}\right)^{(n+1) \varepsilon},
$$

where

$$
B_{n}(\varepsilon)=e^{\varepsilon \gamma_{E}} \frac{\Gamma(1-\varepsilon)}{\Gamma(1+n \varepsilon)} \frac{\Gamma(1+\varepsilon+n \varepsilon) \Gamma(1-\varepsilon-n \varepsilon)}{\Gamma(1-2 \varepsilon-n \varepsilon)} .
$$

Using this we can write the action of the soft Hamiltonian (2.12) on any monomial $(m \geq 0)$ :

$$
\hat{H}_{s} \xi^{m}=\frac{\left(C_{A}-\mathbf{T}_{t}^{2}\right)}{2 \varepsilon}\left(\xi^{m}-\xi^{m+1}\left[1-\hat{B}_{m}(\varepsilon) \frac{2 C_{A}-\mathbf{T}_{t}^{2}}{C_{A}-\mathbf{T}_{t}^{2}}\right]\right)
$$

where we have introduced the loop functions

$$
\hat{B}_{n}(\varepsilon)=1-\frac{B_{n}(\varepsilon)}{B_{0}(\varepsilon)}=2 n(2+n) \zeta_{3} \varepsilon^{3}+3 n(2+n) \zeta_{4} \varepsilon^{4}+\ldots
$$

By making use of eq. (2.16), it can be proven (see [13] for more details) that the wavefunction at order $(\ell-1)$ admits a solution

$$
\Omega_{s}^{(\ell-1)}(p, k)=\frac{\left(C_{A}-\mathbf{T}_{t}^{2}\right)^{\ell-1}}{(2 \varepsilon)^{\ell-1}} \sum_{n=0}^{\ell-1}(-1)^{n}\left(\begin{array}{c}
\ell-1 \\
n
\end{array}\right)\left(\frac{p^{2}}{k^{2}}\right)^{n \varepsilon} \prod_{m=0}^{n-1}\left\{1-\hat{B}_{m}(\varepsilon) \frac{2 C_{A}-\mathbf{T}_{t}^{2}}{C_{A}-\mathbf{T}_{t}^{2}}\right\} .
$$


It is then straightforward to obtain the amplitude, which in the soft approximation reads

$$
\hat{\mathscr{M}}_{\mathrm{NLL}}^{(+, \ell)}=-\frac{i \pi\left(B_{0}\right)^{\ell-1}}{(\ell-1) !} \frac{e^{\varepsilon \gamma_{E}}}{\Gamma(1-\varepsilon)} \int_{0}^{p^{2}} \frac{\mathrm{d} k^{2}}{2 k^{2}}\left(\frac{p^{2}}{k^{2}}\right)^{\varepsilon} \Omega_{s}^{(\ell-1)}(p, k) \mathbf{T}_{s-u}^{2} \mathscr{M}^{(\text {tree })}+\mathscr{O}\left(\varepsilon^{0}\right) .
$$

The last integration can be performed easily, and we arrive at

$$
\begin{aligned}
&\left.\hat{\mathscr{M}}_{\mathrm{NLL}}^{(+, \ell)}\right|_{s}=i \pi \frac{1}{(2 \varepsilon)^{\ell}} \frac{B_{0}^{\ell}(\varepsilon)}{\ell !}\left(1-\hat{B}_{-1}\right)\left(C_{A}-\mathbf{T}_{t}^{2}\right)^{\ell-1} \sum_{n=1}^{\ell}(-1)^{n+1}\left(\begin{array}{l}
\ell \\
n
\end{array}\right) \\
& \times \prod_{m=0}^{n-2}\left[1-\hat{B}_{m}(\varepsilon) \frac{2 C_{A}-\mathbf{T}_{t}^{2}}{C_{A}-\mathbf{T}_{t}^{2}}\right] \mathbf{T}_{s-u}^{2} \mathscr{M}^{(\text {tree })}+\mathscr{O}\left(\varepsilon^{0}\right) .
\end{aligned}
$$

This result looks rather involved, but one must keep in mind that, upon expansion in $\varepsilon$, it contains many finite terms which do not represent the actual amplitude, since we are working in the soft approximation. Given the overall factor of $1 /(2 \varepsilon)^{\ell}$ in eq. (2.19), all the singularities are obtained by retaining only contributions up to $\varepsilon^{\ell-1}$ in the subsequent factors. Eq. (2.19) then can be put in the form

$$
\left.\hat{\mathscr{M}}_{\mathrm{NLL}}^{(+, \ell)}\right|_{s}=i \pi \frac{1}{(2 \varepsilon)^{\ell}} \frac{B_{0}^{\ell}(\varepsilon)}{\ell !}\left(1-R(\varepsilon) \frac{C_{A}}{C_{A}-\mathbf{T}_{t}^{2}}\right)^{-1}\left(C_{A}-\mathbf{T}_{t}^{2}\right)^{\ell-1} \mathbf{T}_{s-u}^{2} \mathscr{M}^{(\text {tree })}+\mathscr{O}\left(\varepsilon^{0}\right),
$$

where we have introduced the function

$$
R(\varepsilon) \equiv \frac{B_{0}(\varepsilon)}{B_{-1}(\varepsilon)}-1=\frac{\Gamma^{3}(1-\varepsilon) \Gamma(1+\varepsilon)}{\Gamma(1-2 \varepsilon)}-1=-2 \zeta_{3} \varepsilon^{3}-3 \zeta_{4} \varepsilon^{4}-6 \zeta_{5} \varepsilon^{5}+\mathscr{O}\left(\varepsilon^{6}\right) .
$$

Notice also that, compared to eq. (2.19), in eq. (2.20) we have rearranged the color structure in such a way to single out the colour structures $C_{A}$ and $\left(C_{A}-\mathbf{T}_{t}^{2}\right)$. This is useful for comparison with the structure of infrared divergences dictated by the soft anomalous dimension, given that the dipole formula of infrared divergencies fixes the singularities of the even amplitude in the highenergy limit to be proportional to the colour structure $\left(C_{A}-\mathbf{T}_{t}^{2}\right)^{\ell-1} \mathbf{T}_{s-u}^{2}$ at $\ell$ loops. By resumming eq. (2.20) according to eq. (2.7) we then get the following closed-form expression for the all-order amplitude:

$$
\begin{aligned}
\left.\hat{\mathscr{M}}_{\mathrm{NLL}}^{(+)}\right|_{s}=\frac{i \pi}{L\left(C_{A}-\mathbf{T}_{t}^{2}\right)}(1 & \left.-R(\varepsilon) \frac{C_{A}}{C_{A}-\mathbf{T}_{t}^{2}}\right)^{-1} \\
\times & {\left[\exp \left\{\frac{B_{0}(\varepsilon)}{2 \varepsilon} \frac{\alpha_{s}}{\pi} L\left(C_{A}-\mathbf{T}_{t}^{2}\right)\right\}-1\right] \mathbf{T}_{s-u}^{2} \mathscr{M}^{(\text {tree })}+\mathscr{O}\left(\varepsilon^{0}\right) . }
\end{aligned}
$$

\section{Infrared singularities from the two-Reggeon cut to all orders}

The result in eq. (2.22) gives the even amplitude at next-to-leading logarithmic accuracy for two parton scattering to all orders in perturbation theory, providing information on infrared divergences to all orders. In what follows we contrast it with what is known about the infrared singularity structure of these amplitudes. 
It is well known that infrared divergences exponentiate in terms of the so-called soft anomalous dimension: this is a consequence of a factorisation theorem, which states that infrared divergences can be absorbed into a multiplicative "infared renormalization" factor $\mathbf{Z}$,

$$
\mathscr{M}\left(\left\{p_{i}\right\}, \mu, \alpha_{s}(\mu)\right)=\mathbf{Z}\left(\left\{p_{i}\right\}, \mu, \alpha_{s}(\mu)\right) \mathscr{H}\left(\left\{p_{i}\right\}, \mu, \alpha_{s}(\mu)\right),
$$

where $\mathscr{H}$ is a finite hard-scattering amplitude while $\mathbf{Z}$ captures all singularities. $\mathbf{Z}$ admits a renormalization group equation whose solution (in the minimal-subtraction scheme) can be written as a path-ordered exponential of the soft anomalous dimension:

$$
\mathbf{Z}\left(\left\{p_{i}\right\}, \mu, \alpha_{s}(\mu)\right)=\mathscr{P} \exp \left\{-\int_{0}^{\mu} \frac{\mathrm{d} \lambda}{\lambda} \Gamma\left(\left\{p_{i}\right\}, \lambda, \alpha_{s}(\lambda)\right)\right\}
$$

The scale dependence of the soft anomalous dimension $\Gamma\left(\left\{p_{i}\right\}, \lambda, \alpha_{s}\right)$ for massless-parton $\left(p_{i}^{2}=0\right)$ scattering is both explicit and via the $4-2 \varepsilon$ dimensional coupling, which to leading order runs as $\alpha_{s}(\mu)=\alpha_{s}(p)\left(p^{2} / \mu^{2}\right)^{\varepsilon}$. The explicit dependence on the scale ( $\Gamma$ is linear in $\log \lambda$ ) reflects the presence of double poles due to overlapping soft and collinear divergences.

To identify the contribution of the soft anomalous dimension in two-parton scattering, $i j \rightarrow i j$, at increasing logarithmic accuracy, let us expand $\Gamma$ in powers of $\alpha_{s}$, keeping the product $\alpha_{s} L$ fixed, as follows:

$$
\Gamma\left(\alpha_{s}(\lambda)\right)=\Gamma_{\mathrm{LL}}\left(\alpha_{s}(\lambda), L\right)+\Gamma_{\mathrm{NLL}}\left(\alpha_{s}(\lambda), L\right)+\Gamma_{\mathrm{NNLL}}\left(\alpha_{s}(\lambda), L\right)+\ldots
$$

The $\mathrm{N}^{k} \mathrm{LL}$ term in eq. (3.3) can be written as an expansion in $\alpha_{s}^{m} L^{m-k}$ for $m \geq 1$. Using Reggepole factorisation it can be shown $[16,18]$ that the leading logarithmic contribution $\Gamma_{\mathrm{LL}}$ takes the one-loop exact form,

$$
\Gamma_{\mathrm{LL}}\left(\alpha_{s}(\lambda)\right)=\frac{\alpha_{s}(\lambda)}{\pi} \frac{\gamma_{K}^{(1)}}{2} L \mathbf{T}_{t}^{2}=\frac{\alpha_{s}(\lambda)}{\pi} L \mathbf{T}_{t}^{2}
$$

This exactly corresponds to the infrared-divergent part of the one-loop gluon Regge trajectory. Note that the LL anomalous dimension has even signature $\Gamma_{\mathrm{LL}}=\Gamma_{\mathrm{LL}}^{(+)}$. At NLL the anomalous dimension can be divided into signature-even and odd parts:

$$
\Gamma_{\mathrm{NLL}}=\Gamma_{\mathrm{NLL}}^{(+)}+\Gamma_{\mathrm{NLL}}^{(-)}
$$

The even part $^{1}$, which is governed by the Regge pole, is two-loop exact, and we refer to [13] for more details. The odd part is sensitive the two-Reggeon cut. At one-loop it can be obtained from the dipole formula $[16,18]$,

$$
\Gamma_{\mathrm{NLL}}^{(-)}=i \pi \frac{\alpha_{s}(\lambda)}{\pi} \mathbf{T}_{s-u}^{2}+O\left(\alpha_{s}^{4} L^{3}\right)
$$

higher order terms were previously unknown; we have presented them for the first time in [13].

The reduced amplitude obtained in section 2 contains information on the infrared divergences of next-to-leading high-energy logarithms to all orders in $\alpha_{s}$, and hence allows us to determine

\footnotetext{
${ }^{1}$ Note that the even part of the NLL anomalous dimension, $\Gamma_{\mathrm{NLL}}^{(+)}$, contributes to the odd NLL amplitude, $\mathscr{M}_{\mathrm{NLL}}^{(-)}$, since it acts on the LL part of $\mathscr{H}$ in eq. (3.1), which is itself odd.
} 
$\Gamma_{\mathrm{NLL}}^{(-)}$to all orders. This can be done by deriving an expression for the amplitude in eq. (2.22) in terms of the soft anomalous dimension. It can be shown (see [13] for a detailed derivation) that this expression is given by

$$
\begin{aligned}
& \exp \left\{\frac{1-B_{0}(\varepsilon)}{2 \varepsilon} \frac{\alpha_{s}}{\pi} L\left(C_{A}-\mathbf{T}_{t}^{2}\right)\right\} \hat{\mathscr{M}}_{\mathrm{NLL}} \\
& =-\int_{0}^{p} \frac{d \lambda}{\lambda} \exp \left\{\frac{1}{2 \varepsilon} \frac{\alpha_{s}(p)}{\pi} L\left(C_{A}-\mathbf{T}_{t}^{2}\right)\left[1-\left(\frac{p^{2}}{\lambda^{2}}\right)^{\varepsilon}\right]\right\} \Gamma_{\mathrm{NLL}}^{(-)}\left(\alpha_{s}(\lambda)\right) \mathscr{M}^{(\text {tree })}+\mathscr{O}\left(\varepsilon^{0}\right) .
\end{aligned}
$$

In minimal subtraction schemes, anomalous dimensions can be extracted by taking the coefficient of pure $1 / \varepsilon$ single poles. Consistency with the infrared factorisation theorem requires then that higher-order poles in eq. (3.1) are correctly reproduced, once the anomalous dimension has been determined. We find (see [13] for more details)

$$
\Gamma_{\mathrm{NLL}}^{(-, \ell)}=\left.\frac{i \pi}{(\ell-1) !}\left(1-R\left(\frac{x}{2}\left(C_{A}-\mathbf{T}_{t}^{2}\right)\right) \frac{C_{A}}{C_{A}-\mathbf{T}_{t}^{2}}\right)^{-1}\right|_{x^{\ell-1}} \mathbf{T}_{s-u}^{2} .
$$

where the function $R(\varepsilon)=-2 \zeta_{3} \varepsilon^{3}+\ldots$ is defined in eq. (2.21). Equation (3.8) gives the soft anomalous dimension in the Regge limit to any loop order at next-to-leading logarithmic accuracy (i.e. all terms of the form $\alpha_{s}^{\ell} L^{\ell-1}$ ). In other words, we now know eq. (3.6) to all orders:

$$
\Gamma_{\mathrm{NLL}}^{(-)}=\sum_{\ell=1}^{\infty} \Gamma_{\mathrm{NLL}}^{(-, \ell)}\left(\frac{\alpha_{s}(\lambda)}{\pi}\right)^{\ell} L^{\ell-1} .
$$

It may be useful to expand the above formula explicitly for the first few orders, in order to visualise better the prediscted structure of infrared divergences:

$$
\begin{array}{ll}
\Gamma_{\mathrm{NLL}}^{(-, 1)} & =i \pi \mathbf{T}_{s-u}^{2}, \quad \quad \Gamma_{\mathrm{NLL}}^{(-, 2)}=0, \\
\Gamma_{\mathrm{NLL}}^{(-, 4)} & =-i \pi \frac{\zeta_{3}}{24} C_{A}\left(C_{A}-\mathbf{T}_{t}^{2}\right)^{2} \mathbf{T}_{s-u}^{2}, \\
\Gamma_{\mathrm{NLL}}^{(-, 5)} & =-i \pi \frac{\zeta_{4}}{128} C_{A}\left(C_{A}-\mathbf{T}_{t}^{2}\right)^{3} \mathbf{T}_{s-u}^{2}, \\
\Gamma_{\mathrm{NLL}}^{(-, 6)} & =-i \pi \frac{\zeta_{5}}{640} C_{A}\left(C_{A}-\mathbf{T}_{t}^{2}\right)^{4} \mathbf{T}_{s-u}^{2}, \\
\Gamma_{\mathrm{NLL}}^{(-, 7)} & =i \pi \frac{1}{720}\left[\frac{\zeta_{3}^{2}}{16} C_{A}^{2}\left(C_{A}-\mathbf{T}_{t}^{2}\right)^{4}+\frac{1}{32}\left(\zeta_{3}^{2}-5 \zeta_{6}\right) C_{A}\left(C_{A}-\mathbf{T}_{t}^{2}\right)^{5}\right] \mathbf{T}_{s-u}^{2} .
\end{array}
$$

It may be interesting to note that infrared divergences proportional to a new color structure arise every three loops. These results are valid in any gauge theory, and hold modulo colour operators which vanish when acting on the Regge limit of the tree amplitude (which is given by the $t$-channel gluon exchange diagram).

It is interesting to study the convergence properties and the asymptotic high-energy behaviour of the soft anomalous dimension in eq. (3.8). To this end it is useful to specify the relevant colour charge exchanged in the $t$ channel, $\mathbf{T}_{t}^{2}$. Focusing on gluon-gluon scattering, the $t$-channel colour flow can be any of the $\mathrm{SU}\left(N_{c}\right)$ representations appearing in eq. (1.2). As explained, only even 
representations are relevant for the two-Reggeon amplitude discussed here, i.e. the singlet, where $\mathbf{T}_{t}^{2}=0$, the symmetric octet, with $\mathbf{T}_{t}^{2}=C_{A}=N_{c}$, and the 27 representation, with $\mathbf{T}_{t}^{2}=2\left(N_{c}+1\right)$.

It is easy to realise that the symmetric octet constitutes a trivial solution to eq. (2.9), with no corrections to the reduced amplitude beyond one loop, given that $\mathbf{T}_{t}^{2}=C_{A}$. The reduced amplitude for the symmetric octet state is thus one-loop exact, corresponding to a simple Regge-pole behaviour with a gluon Regge trajectory for the original amplitude according to eq. (2.2). This of course reproduces the known behaviour of the symmetric-octet exchange used in the original derivation of the BFKL equation. The singlet - the famous Pomeron - and 27 representation have instead non-trivial radiative corrections associated with a Regge cut; we therefore study the convergence properties of the series in eq. (3.9) for these two representations. One immediately notes that this series is highly convergent due to the $1 /(\ell-1)$ ! prefactor in eq. (3.8). We can go further and establish that the anomalous dimension eq. (3.8) has an infinite radius of convergence as a function of $x \equiv L \alpha_{s} / \pi$. To see this we write the resummed soft anomalous dimension as:

$$
\Gamma_{\mathrm{NLL}}^{(-)}=i \pi \frac{\alpha_{s}}{\pi} G\left(\frac{\alpha_{s}}{\pi} L\right) \mathbf{T}_{s-u}^{2},
$$

and it can be shown that the function $G(x)$ can be written as the Borel transform of a function $g(y)$ :

$$
G(x)=\frac{1}{2 \pi i} \int_{w-i \infty}^{w+i \infty} d \eta g\left(\frac{1}{\eta}\right) \mathrm{e}^{\eta x},
$$

where the integration contour runs parallel to the imaginary axis, to the right of all singularities of the integrand, and

$$
g(y)=y\left(1-R\left(\frac{y}{2}\left(C_{A}-\mathbf{T}_{t}^{2}\right)\right) \frac{C_{A}}{C_{A}-\mathbf{T}_{t}^{2}}\right)^{-1} .
$$

The function $g(y)$ has only isolated poles away from the origin and has a finite radius of convergence: it is well-defined in a disc around the origin. It then follows that $G(x)$ has an infinite radius of convergence, hence this function - and the soft anomalous dimension $\Gamma_{\mathrm{NLL}}^{(-)}$in eq. (3.11) - is an entire function, free of any singularities for any finite $x=L \alpha_{s} / \pi$.

Evaluating the integral (3.12) and plotting $G(x)$ for larger values of $x$ reveals oscillations with a constant period and an exponentially growing amplitude. Since this behaviour is difficult to capture graphically we instead show the logarithm of $|G(x)|$ weighted by the sign of $G(x)$ in figure 3. This observation suggests to approximate (3.12) by

$$
G(x) \rightarrow c e^{a x} \cos (b x+d),
$$

for sufficiently large values of $x$. It can be shown that this is equivalent to

$$
g\left(\frac{1}{\eta}\right) \rightarrow c \operatorname{Re}\left[\frac{e^{i d}}{\eta-a-i b}\right]=\frac{c}{2}\left(\frac{e^{i d}}{\eta-a-i b}+\frac{e^{-i d}}{\eta-a+i b}\right),
$$

which is to be integrated as in (3.12) with a contour to the right of the poles. We thus find that to capture the behaviour $G(x)$ at large $x$ it is sufficient to simply consider $g\left(\frac{1}{\eta}\right)$ as a pair of complexconjugated poles at $\eta=a \pm i b$. Indeed, numerically extracting the rightmost poles of $g\left(\frac{1}{\eta}\right)$ of eq. (3.13) to identify the parameters $a$ and $b$ in eq. (3.15), and dividing the full, numericallyevaluated, $G(x)$ by $e^{a x}$ leaves us with almost pure cosine-like behaviour for any $x \gg 1$. For reference, we quote our numerical results for $a, b, c$ and $d$ in table 1. 


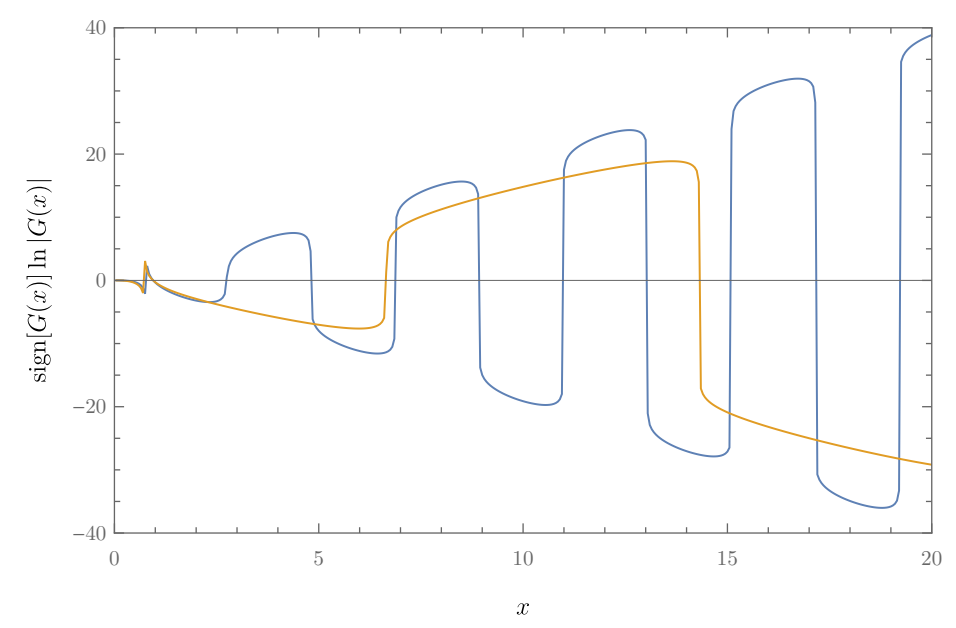

Figure 3: Numerical results for sign $[G(x)] \ln |G(x)|$ for the singlet (blue) and 27 exchange (orange). The "heartbeat" at small $x$ reflects the logarithmic divergence of $\ln |G(x)|$ where $G(x)$ changes its sign for the first time (similar divergences occur every oscillation but are not visible due to the finite resolution of the plot).

\begin{tabular}{|c|c|c|c|c|}
\hline & $a$ & $b$ & $c$ & $d$ \\
\hline 1 & 1.97 & 1.52 & 0.25 & 0.48 \\
27 & 1.46 & 0.41 & 0.58 & 2.01 \\
\hline
\end{tabular}

Table 1: Numerical results for $a, b, c$ and $d$, cf. eq. (3.14), for the singlet (1) and 27 representation.

\section{Conclusion}

We considered the even signature component of two-to-two parton scattering amplitudes in the high-energy limit. This amplitude is dominated by the $t$-channel exchange of a state consisting of two reggeized gluons, corresponding to the simplest example of a Regge cut in QCD. The amplitude can be evaluated in QCD perturbation theory by iteratively solving the BFKL equation. Each order in perturbation theory corresponds to one additional rung in the BFKL ladder, building up a tower of so-called next-to-leading logarithms, $\mathscr{O}\left(\alpha_{s}^{\ell} L^{\ell-1}\right)$. Although the BFKL Hamiltonian has been diagonalised in many cases [4], the dimensionally-regulated Hamiltonian relevant for partonic amplitudes has remained more difficult to handle.

Our first observation was that the wavefunction describing the two reggeized gluons remains finite through BFKL evolution for any number of rungs, while the corresponding amplitude develops infrared singularities due to the soft limit of the wavefunction. We further observed that the evolution of a state in which one of the two reggeized gluons is much softer than the other, yields again a similar state. In other words, the soft approximation is consistent with BFKL evolution, and as a consequence, one can systematically solve the equation to any loop order within this approximation. We found that the soft approximation leads to a major simplification, where all integrals reduce to products of bubbles, and the wavefunction at any given order is simply a polynomial of that order in $\left(p^{2} / k^{2}\right)^{\varepsilon}$. This eventually allowed us to determine the singularities of the amplitude in a closed form to any order, as given in eq. (2.22).

At the next step we contrasted the singularity structure we obtained though BFKL evolution 
with the known exponentiation properties of infrared singularities. As expected, we found that the two are consistent, and this provides a highly non-trivial check of the calculation. The leading singularity at each order, $\mathscr{O}\left(\alpha_{s}^{\ell} L^{\ell-1} / \varepsilon^{\ell}\right)$, is simply related to the one-loop soft anomalous dimension, and has a colour structure proportional to $\left(C_{A}-\mathbf{T}_{t}^{2}\right)^{\ell-1}$. New singularities, with fewer powers of $1 / \varepsilon$ and different colour structures, appear starting from four loop. These correspond to new terms in the imaginary part of the soft anomalous dimension, eq. (3.10). We were thus able to determine the soft anomalous dimension at next-to-leading logarithmic accuracy in the high-energy limit to all orders. These results also provide a valuable input for determining the structure of long-distance singularities for general kinematics using a bootstrap approach, as done at the three-loop order in ref. [27].

\section{References}

[1] E. A. Kuraev, L. N. Lipatov and V. S. Fadin, Sov. Phys. JETP 45 (1977) 199 [Zh. Eksp. Teor. Fiz. 72 (1977) 377].

[2] I. I. Balitsky and L. N. Lipatov, Sov. J. Nucl. Phys. 28 (1978) 822 [Yad. Fiz. 28 (1978) 1597].

[3] P. D. B. Collins, "An Introduction to Regge Theory and High-Energy Physics", Cambridge Monographs on Mathematical Physics, Cambridge Univ. Press, Cambridge, 1977, doi:10.1017/CBO9780511897603

[4] L. N. Lipatov, Sov. Phys. JETP 63 (1986) 904 [Zh. Eksp. Teor. Fiz. 90 (1986) 1536].

[5] F. E. Low, Phys. Rev. D 12 (1975) 163. doi:10.1103/PhysRevD.12.163

[6] S. Nussinov, Phys. Rev. Lett. 34 (1975) 1286. doi:10.1103/PhysRevLett.34.1286

[7] J. F. Gunion and D. E. Soper, Phys. Rev. D 15 (1977) 2617. doi:10.1103/PhysRevD.15.2617

[8] L. N. Lipatov, JETP Lett. 59 (1994) 596 [Pisma Zh. Eksp. Teor. Fiz. 59 (1994) 571] [hep-th/9311037].

[9] L. D. Faddeev and G. P. Korchemsky, Phys. Lett. B 342 (1995) 311 doi:10.1016/0370-2693(94)01363-H [hep-th/9404173].

[10] Y. L. Dokshitzer and G. Marchesini, JHEP 0601 (2006) 007 doi:10.1088/1126-6708/2006/01/007 [hep-ph/0509078].

[11] S. Caron-Huot, JHEP 1505 (2015) 093 doi:10.1007/JHEP05(2015)093 [arXiv:1309.6521 [hep-th]].

[12] S. Caron-Huot, E. Gardi and L. Vernazza, JHEP 1706 (2017) 016 doi:10.1007/JHEP06(2017)016 [arXiv:1701.05241 [hep-ph]].

[13] S. Caron-Huot, E. Gardi, J. Reichel and L. Vernazza, JHEP 1803 (2018) 098 doi:10.1007/JHEP03(2018)098 [arXiv:1711.04850 [hep-ph]].

[14] V. Del Duca, G. Falcioni, L. Magnea and L. Vernazza, Phys. Lett. B 732 (2014) 233 doi:10.1016/j.physletb.2014.03.033 [arXiv:1311.0304 [hep-ph]].

[15] V. Del Duca, G. Falcioni, L. Magnea and L. Vernazza, JHEP 1502 (2015) 029 doi:10.1007/JHEP02(2015)029 [arXiv:1409.8330 [hep-ph]].

[16] V. Del Duca, C. Duhr, E. Gardi, L. Magnea and C. D. White, Phys. Rev. D 85 (2012) 071104 doi:10.1103/PhysRevD.85.071104 [arXiv:1108.5947 [hep-ph]]. 
[17] G. P. Korchemsky, Phys. Lett. B 325 (1994) 459 doi:10.1016/0370-2693(94)90040-X [hep-ph/9311294].

[18] V. Del Duca, C. Duhr, E. Gardi, L. Magnea and C. D. White, JHEP 1112 (2011) 021 doi:10.1007/JHEP12(2011)021 [arXiv:1109.3581 [hep-ph]].

[19] S. Catani, Phys. Lett. B 427 (1998) 161 doi:10.1016/S0370-2693(98)00332-3 [hep-ph/9802439].

[20] S. M. Aybat, L. J. Dixon and G. F. Sterman, Phys. Rev. D 74 (2006) 074004 doi:10.1103/PhysRevD.74.074004 [hep-ph/0607309].

[21] E. Gardi and L. Magnea, JHEP 0903 (2009) 079 doi:10.1088/1126-6708/2009/03/079 [arXiv:0901.1091 [hep-ph]].

[22] T. Becher and M. Neubert, JHEP 0906 (2009) 081 Erratum: [JHEP 1311 (2013) 024] doi:10.1088/1126-6708/2009/06/081, 10.1007/JHEP11(2013)024 [arXiv:0903.1126 [hep-ph]].

[23] L. J. Dixon, E. Gardi and L. Magnea, JHEP 1002 (2010) 081 doi:10.1007/JHEP02(2010)081 [arXiv:0910.3653 [hep-ph]].

[24] V. Ahrens, M. Neubert and L. Vernazza, JHEP 1209 (2012) 138 doi:10.1007/JHEP09(2012)138 [arXiv:1208.4847 [hep-ph]].

[25] Ø. Almelid, C. Duhr and E. Gardi, Phys. Rev. Lett. 117 (2016) no.17, 172002 doi:10.1103/PhysRevLett.117.172002 [arXiv:1507.00047 [hep-ph]].

[26] E. Gardi, Ø. Almelid and C. Duhr, PoS LL 2016 (2016) 058 doi:10.22323/1.260.0058 [arXiv:1606.05697 [hep-ph]].

[27] Ø. Almelid, C. Duhr, E. Gardi, A. McLeod and C. D. White, JHEP 1709 (2017) 073 doi:10.1007/JHEP09(2017)073 [arXiv:1706.10162 [hep-ph]]. 